\title{
Structural Characterization of Bimetallic Nanocrystal Electrocatalysts
}

\author{
Brian T. Sneed, ${ }^{1}$ Chun-Hong Kuo, ${ }^{2}$ and David A. Cullen ${ }^{3}$ \\ 1. Center for Nanophase Materials Sciences, Oak Ridge National Laboratory, Oak Ridge, TN, USA \\ 2. Institute of Chemistry, Academia Sinica, Taipei, Taiwan \\ 3. Materials Science and Technology Division, Oak Ridge National Laboratory, Oak Ridge, TN, USA
}

Late transition metal nanocrystals find applications in heterogeneous catalysis such as plasmonenhanced catalysis and as electrode materials for fuel cells, a zero-emission and sustainable energy technology. Their commercial viability for automotive transportation has steadily increased in recent years, almost exclusively due to the discovery of more efficient bimetallic nanocatalysts for the oxygen reduction reaction (ORR) at the cathode. Despite improvements to catalyst design, achieving high activity while maintaining durability is essential to further enhance their performance for this and other important applications in catalysis. Electronic effects arising from the generation of metal-metal interfaces, from plasmonic metals, and from lattice distortions, can vastly improve sorption properties at catalytic surfaces, while increasing durability.[1] Multimetallic lattice-strained nanoparticles are thus an interesting opportunity for fundamental research.[2,3]

A colloidal synthesis approach is demonstrated to produce AuPd alloy and $\mathrm{Pd} @ \mathrm{Au}$ core-shell nanoicosahedra as catalysts for electro-oxidations. The nanoparticles are characterized using aberrationcorrected scanning transmission electron microscopy (ac-STEM) and large solid angle energy dispersive X-ray spectroscopy (EDS) on an FEI Talos 4-detector STEM/EDS system. Figure 1 shows bright-field (BF) and high-angle annular dark-field (HAADF) ac-STEM images of the alloy and core-shell nanoicosahedra together with EDS line-scans and elemental maps. These structures are unique in that the presence of twin boundaries, alloying, and core-shell morphology could create highly strained surfaces and interfaces. The shell thickness of the core-shell structures observed in HAADF-STEM images is tuned by adjusting the ratio between metal precursors (Figure 2a-f) to produce shells ranging from a few to several monolayers. Specific activity was measured in ethanol electro-oxidation to examine the effect of shell thickness on catalytic activity. A volcano relationship was observed for the core-shell nanoicosahedra having different Pd-shell thicknesses as Pd content is increased (Figure 2g).

Durability tests are ongoing for the AuPd system; however, promising ORR materials and morphologies have also been synthesized for a more cost-effective $\mathrm{Cu}$-based system of $\mathrm{Cu}-\mathrm{CuM}(\mathrm{M}=\mathrm{Pd}, \mathrm{Rh}, \mathrm{Pt})$ core-alloy-shell nanocrystals. The synthesis, characterization, and catalytic behavior of different highindex faceted morphologies of $\mathrm{Cu}$-based materials towards ORR and methanol oxidation catalysis will be discussed, where we show how they exceed the performance of commercial Pd- and Pt- based catalysts. The development of new materials and their characterization is critical to understanding the effects of structure and composition on catalysis. Future efforts are directed at resolving these structures and more industrially relevant fuel cell catalysts in 3D through electron tomography.[4]

References:

[1] X. Huang, et al., Science 348 (2015) p. 1230.

[2] P. Strasser, et al., Nat. Chem. 2 (2010) p. 454.

[3] C. Chen, et al., Science 343 (2014) p. 1339. 
[4] Microscopy performed as part of a user project through ORNL's Center for Nanophase Materials Sciences, which is a U.S. DOE Office of Science User Facility, and instrumentation provided by the U.S. DOE Office of Nuclear Energy, Fuel Cycle R\&D Program, and the Nuclear Science User Facilities.
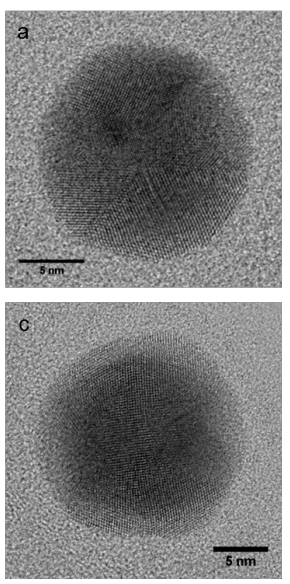
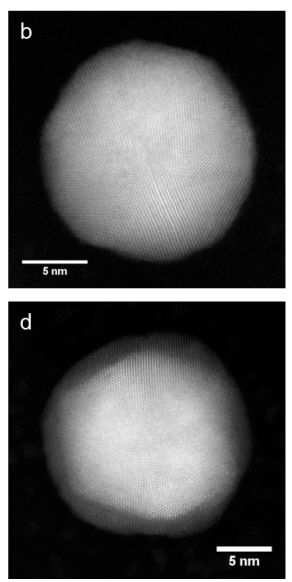
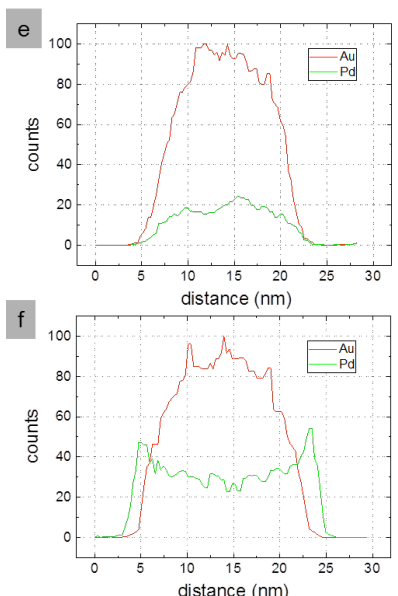
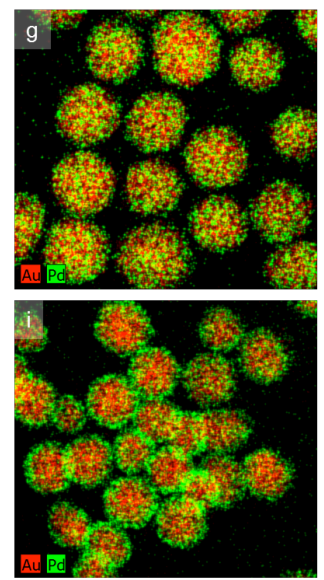
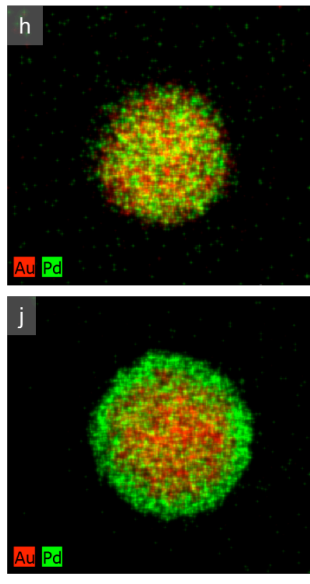

Figure 1. ac-STEM BF/HAADF images, EDS line-scans, and elemental maps. BF/HAADF image pairs of icosahedral $(a, b)$ AuPd alloy and $(c, d)$ Pd@Au core-shell nanoparticles. STEM/EDS maps and line-scans of alloy and core-shell AuPd nanoicosahedra: (e) EDS line-scan across the center of an AuPd alloy nanoicosahedron and (f) line-scan across the center of a Pd@Au core-shell nanoicosahedron, and overlayed Au and Pd EDS maps of $(\mathrm{a}, \mathrm{b})$ alloy and $(\mathrm{c}, \mathrm{d})$ core-shell icosahedra.
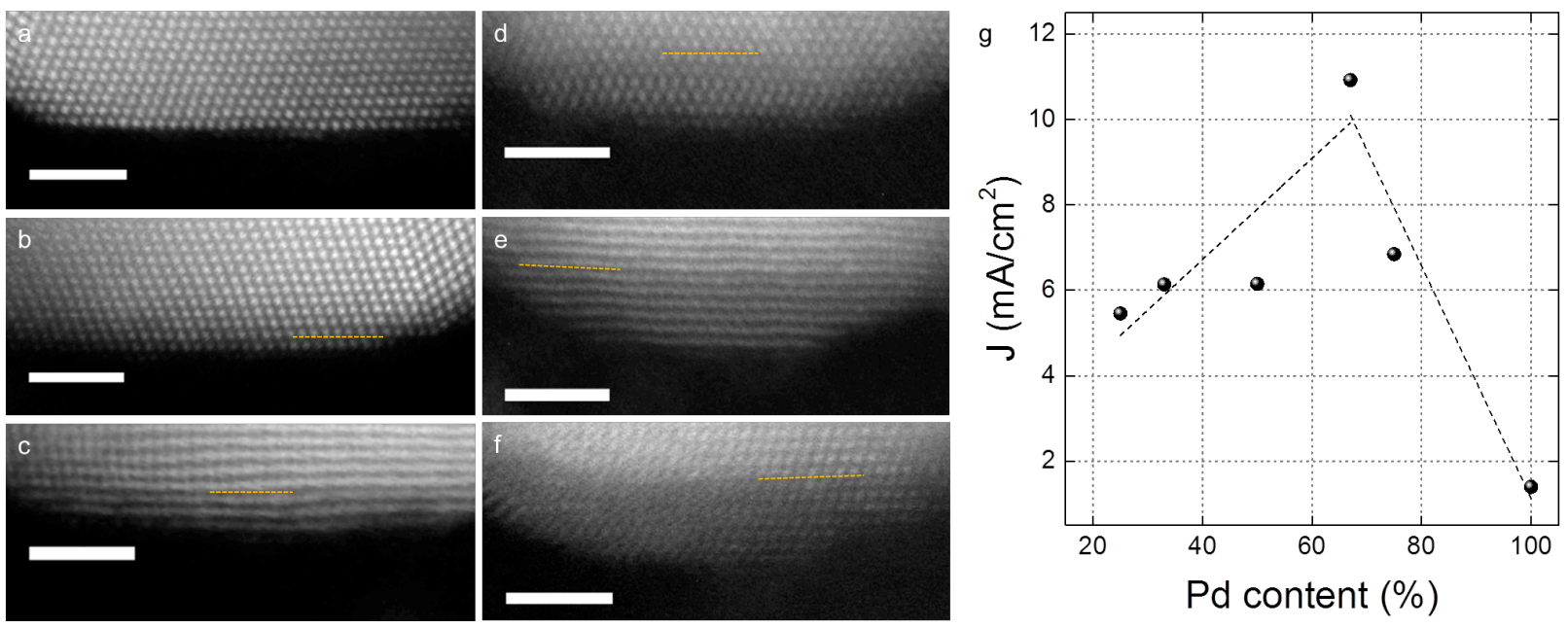

Figure 2. (a-f) HAADF-STEM images of Pd-shells with increasing thickness from a monolayer to several layers by adjusting the ratio of metals in the synthesis (lines mark the interface). (g) Plot of specific activity for electrochemical oxidation of ethanol vs. Pd content in nanoparticle. 